\title{
REFERENCE
}

1. Raychaudhuri S, Remmers EF, Lee AT, et al. Common variants at CD40 and other loci confer risk of rheumatoid arthritis. Nat Genet 2008;40:1216-23.

\section{A195 GENETIC INTERACTION IN THE SUSCEPTIBILITY OF RHEUMATOID ARTHRITIS}

Nina A Daha, ${ }^{1}$ Annemiek Willemze, ${ }^{1}$ David B Robinson, ${ }^{2}$ Kiem G Oen, ${ }^{2}$ Irene Smolik, ${ }^{2}$ Donna Hart, ${ }^{2}$ Wendimagegn Ghidey, Jeanine J Houwing-Duistermaat, ${ }^{1}$ KA Siminovitch, ${ }^{3}$ Tom WJ Huizinga, ${ }^{1}$ Hani S El-Gabalawy, ${ }^{2}$ René EM Toes ${ }^{1}$ Leiden University Medical Center, Leiden, Netherlands, '2University of Manitoba, Winnipeg, Manitoba, Canada; ${ }^{3}$ University of Toronto, Toronto, Canada

10.1136/ard.2010.149021.5

Introduction Recent advances have revolutionised the identification of genes underlying common diseases. For rheumatoid arthritis (RA), over 20 genes have been identified to associate to disease susceptibility. The strongest of these genetic associations is with particular genes within the human leucocyte antigen (HLA) region. To date, however, little is known about underlying relations between genes on a genetic level, which provides more insight into biological pathways involved. In the current study the authors investigated this for the strongly associated HLA region in a North American Native population. This population has a high RA prevalence rate with multicase families and, most importantly, a high prevalence of shared epitope alleles in the background population.

Methods RA gene polymorphisms, identified previously, ${ }^{1}$ were genotyped in 333 North American Native RA patients, 295 first degree relatives and 490 unrelated controls. HLADRB1 alleles were tested by sequencing. Allele distribution was analysed using a $\chi^{2}$ test with one degree of freedom and as interaction criteria departure from multiplicative effects was used. Significant associations were tested for replication in a Dutch RA population ( $\mathrm{n}=918)$ and controls $(\mathrm{n}=1309)$.

Results A significant association was detected for MMEL1TNFRSF14 in the Native American population (OR 1.45, 95\% CI 1.09 to $1.94, p=0.009$ ), with a significant association in the shared epitope positive group and not in the shared epitope negative group (OR 1.66, 95\% CI 1.20 to 2.30, $p=0.002$ and OR $0.85,95 \%$ CI 0.47 to $1.55, p=0.60$, respectively). Analysis in the Dutch population demonstrated a similar trend in RA association (shared epitope positive group: OR1.31, 95\% CI 1.0 to $1.70, p=0.04$; shared epitope negative group: OR 1.11, $95 \%$ CI 0.81 to $1.53, p=0.51$ ), but significant statistical evidence for interaction could not be established.

Conclusion In the current study the authors demonstrate a novel interaction between the HLA region and MMEL1TNFRSF14 in a Native American population, with a similar trend in a Dutch population of RA patients. This interaction is particularly important since shared epitope alleles are highly present in the Native American population, yet most individuals stay disease free. This finding might give more insight into biological pathways involved in disease and therefore give rise to future therapeutic targets. 